\title{
SUPPORT POINTS OF SUBORDINATION FAMILIES
}

\author{
D. J. HALLENBECK
}

(Communicated by Irwin Kra)

\begin{abstract}
Let $s(F)$ denote the set of functions subordinate to a function $F$ analytic in the unit disc $\Delta$. Let $H s(F)$ denote the closed convex hull of $s(F)$ and supp $s(F)$ the set of support points of $s(F)$. We prove the following

THEOREM. Let $F$ be analytic in $\Delta$ and satisfy

(1) $H s(F)=\left\{\int_{\partial \Delta} F(x z) d \mu(x): \mu\right.$ a probablity measure on $\left.\partial \Delta\right\}$ and

(2) $F(z)=G(z) /\left(z-x_{0}\right)^{\alpha}$ where $G$ is analytic in $\Delta$, continuous in $\bar{\Delta}$, $G\left(x_{0}\right) \neq 0$ and $\alpha>1$. Then $\operatorname{supp} s(F)=\{F(x z):|x|=1\}$.
\end{abstract}

Introduction. Let $\Delta=\{z:|z|<1\}$ and let $\mathbf{A}$ denote the set of functions analytic in $\Delta$. Let $B_{0}=\{\phi: \phi \in \mathbf{A}$ and $|\phi(z)| \leq|z|\}$. $\mathbf{A}$ is a locally convex linear topological space with respect to the topology given by uniform convergence on compact subsets of $\Delta$. By a continuous, linear functional on $\mathbf{A}$, we mean a complex-valued functional defined on $\mathbf{A}$ that is continuous and linear. In other words, if $J$ is such a functional, then $J(a f+b g)=a J(f)+b J(g)$ whenever $a$ and $b$ belong to $\mathbf{C}$ and $f$ and $g$ belong to $\mathbf{A}$. Also, if $f_{n} \in \mathbf{A}(n=1,2, \ldots)$ and $f_{n} \rightarrow f$, then $J\left(f_{n}\right) \rightarrow J(f)$. Each continuous, linear functional $J$ on $\mathbf{A}$ is given by a sequence $\left\{b_{n}\right\}(n=1,2, \ldots)$ which satisfies $\varlimsup_{n \rightarrow \infty} \sqrt{\left|b_{n}\right|}<1$ and

$$
J(f)=\sum_{n=0}^{\infty} a_{n} b_{n}
$$

where $f(z)=\sum_{n=0} a_{n} z^{n}(|z|<1)[7]$. For such a sequence $\left\{b_{n}\right\}$, the function $F(z)=\sum_{n=0}^{\infty} b_{n} z^{n}$ is analytic in $\bar{\Delta}=\{z:|z| \leq 1\}$.

A function $f$ is called a support point of a compact subset $\mathbf{F}$ of $\mathbf{A}$ if $f \in \mathbf{F}$ and if there is a continuous, linear functional $J$ on $\mathbf{A}$ so that

$$
\operatorname{Re} J(f)=\max \{\operatorname{Re} J(g): g \in \mathbf{F}\}
$$

and $\operatorname{Re} J$ is not constant on $\mathbf{F}$. In general, for a fixed continuous, linear functional $J$, the solution set contains an extreme point of the closed convex hull of $\mathbf{F}$ which we denote by $H \mathbf{F}$. The set of extreme points of a family $\mathbf{F}$, we denote by $E \mathbf{F}$ and the set of support points by supp F.

We recall the definition of subordination between two functions, say $f$ and $F$, analytic in $\Delta$. This means that there is an analytic function $\phi$ so that $\phi \in B_{0}$ and $f=F \cdot \phi$. If $F$ is univalent in $\Delta$, the subordination is equivalent to $f(0)=F(0)$ and $f(\Delta) \subset F(\Delta)$. The set of all functions subordinate to a fixed function $F$ we denote by $s(F)$. We note that $s(F)$ is a compact subset of $\mathbf{A}$ and $s(F)=\left\{F \cdot \phi: \phi \in B_{0}\right\}$.

Received by the editors October 29, 1986 and, in revised form, March 31, 1987.

1980 Mathematics Subject Classification (1985 Revision). Primary 30C80; Secondary 30C70. Key words and phrases. Support points, subordination. 
In Theorem 1 we give conditions that guarantee $\operatorname{supp} s(F)=\{F(x z):|x|=1\}$. Support points of $s(F)$.

THEOREM 1. Let $F$ be analytic in $\Delta$ and satisfy

$$
F(z)=G(z) /\left(z-x_{0}\right)^{\alpha}
$$

where $G$ is analytic in $\Delta$, continuous in $\bar{\Delta}, G\left(x_{0}\right) \neq 0$ and $\alpha>1$.

$$
H s(F)=\left\{\int_{\partial \Delta} F(x z) d \mu(x): \mu \in \Lambda\right\}
$$

where $\Lambda$ is the set of probability measures on $\partial \Delta$. Then $\operatorname{supp} s(F)=\{F(x z):|x|$ $=1\}$.

ProOF. The inclusion $\{F(x z):|x|=1\} \subset \operatorname{supp} s(F)$ is known [6, p. 103] and so we need only to prove that $\operatorname{supp} s(F) \subset\{F(x z):|x|=1\}$.

Suppose that $f \in \operatorname{supp} s(F)$. It follows that $f=F \cdot \phi$ where $\phi \in \operatorname{supp} B_{0}[\mathbf{1}, \mathbf{5}]$. There exists a continuous, linear functional $J$ on $\mathbf{A}$ so that $\operatorname{Re} J$ is nonconstant on $s(F), J$ is given by $\left\{b_{n}\right\}$ as in (1) and (2) holds. Let $F(z)=A_{0}+\sum_{n=1}^{\infty} A_{n} z^{n}$. Then $F(x z)=A_{0}+\sum_{n=1}^{\infty} A_{n} x^{n} z^{n}$ and

$$
J(F(x z))=b_{0} A_{0}+\sum_{n=1}^{\infty} b_{n} A_{n} x^{n}=G(x)
$$

defines a function $G$ analytic in $\bar{\Delta}$, because $\varlimsup_{n \rightarrow \infty} \sqrt{\left|b_{n} A_{n}\right|} \leq \varlimsup_{n \rightarrow \infty} \sqrt{\left|b_{n}\right|}<1$. Since (4) implies that $E H s(F) \subset\{F(x z):|x|=1\}$, it is clear that $G$ is nonconstant, otherwise $\operatorname{Re} J$ is constant on $E H s(F)$ and so on $s(F)$. This is impossible by assumption. If we let $M=\max \{\operatorname{Re} J(g): g \in s(F)\}$, then $\operatorname{Re} G(x)=M$ has only a finite number of solutions with $|x|=1$ [3, p. 106]. By familiar arguments, [3, p. 100], we conclude that the solution set over $H s(F)$ is the convex hull of a finite number of extreme points of $H s(F)$. It follows that

$$
f(z)=\sum_{k=1}^{n} \lambda_{k} F\left(x_{k} z\right)
$$

where $n$ is a positive integer, $\lambda_{k} \geq 0,\left|x_{k}\right|=1(k=1,2, \ldots, n)$ and $\sum_{k=1}^{n} \lambda_{k}=1$. We see from (3) that $f(z)=G(\phi(z)) /\left(\phi(z)-x_{0}\right)^{\alpha}$. Let $w_{k}=\bar{x}_{k} x_{0}(k=1,2, \ldots, n)$ then (3), (6) and the previous equality imply $\phi\left(w_{k}\right)=x_{0}$ for $k=1,2, \ldots, n$. It is known $[4$, p. 83$]$ that we can write

$$
\frac{1}{1-\bar{x}_{0} \phi(z)}=\sum_{k=1}^{m} t_{k} \frac{1}{1-y_{k} z}
$$

where $t_{k} \geq 0,\left|y_{k}\right|=1(k=1,2, \ldots, m)$ and $\sum_{k=1}^{m} t_{k}=1$. It follows that

$$
\sum_{k=1}^{n} \lambda_{k} F\left(x_{k} z\right)=a G(\phi(z))\left[\sum_{k=1}^{m} t_{k} \frac{1}{1-y_{k} z}\right]^{\alpha}
$$

where $a=\left(-x_{0}\right)^{-\alpha}$. By using (3) and comparing singularities on both sides of (8) we conclude that $n=m$ and so we have

$$
\sum_{k=1}^{n} \lambda_{k} F\left(x_{k} z\right)=a G(\phi(z))\left[\sum_{k=1}^{n} t_{k} \frac{1}{1-y_{k} z}\right]^{\alpha}
$$


Using (3) we can rewrite (9) as

$$
a \sum_{k=1}^{n} \lambda_{k} \frac{G\left(x_{k} z\right)}{\left(1-\bar{w}_{k} z\right)^{\alpha}}=a G(\phi(z))\left[\sum_{k=1}^{n} t_{k} \frac{1}{1-y_{k} z}\right]^{\alpha} .
$$

Thus $w_{k}=\bar{y}_{k}$ for $k=1,2, \ldots, n$. If we let $z=r \bar{y}_{j}(j \in\{1,2, \ldots, n\})$, multiply by $(1-r)^{\alpha}$ and let $r \rightarrow 1$ in (10) we obtain

$$
G\left(x_{j} \bar{y}_{j}\right) \lambda_{j}=G\left(\phi\left(\bar{y}_{j}\right)\right) t_{j}^{\alpha} .
$$

But $\phi\left(\bar{y}_{j}\right)=\phi\left(w_{j}\right)=x_{0}=x_{j} w_{j}=x_{j} \bar{y}_{j}$ and $G\left(x_{0}\right) \neq 0$ by (3). Thus (11) implies $t_{j}^{\alpha}=\lambda_{j}$ for $j=1,2, \ldots, n$.

To finish the proof, assume that each $\lambda_{k}$ in (6) satisfies $0<\lambda_{k}<1$. As we showed above $\lambda_{k}=t_{k}^{\alpha}$ for $k=1,2, \ldots, n$. Note that $\alpha>1$ and $0<t_{k}<1$ implies that $t_{k}^{\alpha}<t_{k}$ for $k=1,2, \ldots, n$. Hence, we conclude that $1=\sum_{k=1}^{n} \lambda_{k}=$ $\sum_{k=1}^{n} t_{k}^{\alpha}<\sum_{k=1}^{n} t_{k}=1$. This is a contradiction and so $\lambda_{j}=1$ for some $j$ and $f(z)=F\left(x_{j} z\right)$. This completes the proof.

REMARK. In a recent paper [2], Y. Abu-Muhanna proved that if $F$ is univalent in $\Delta$, the complement of $F(\Delta)$ is convex and $\partial F(\Delta)$ satisfies a smoothness condition at $\infty$ then (3) holds. He also proved that under these assumptions $E H s(F)=$ $\{F(x z):|x|=1\}$ and so (4) also holds. Hence the conclusion of Theorem 1 holds under the assumptions made in [2]. When $F(z)=((1+c z) /(1-z))^{\alpha}$ for $|c| \leq 1$, $c \neq-1$ and $\alpha>1$ it is known that $\operatorname{supp} s(F)=\{F(x z):|x|=1\}$ [5]. This follows directly from Theorem 1 .

COROLlaRY 2. Suppose F satisfies the hypothesis of Theorem 1. Suppose additionally that $F(z) \neq 0$ in $\Delta$ and $\log F(z)$ is convex. Then for $\beta \geq 1, \operatorname{supp} s\left(F^{\beta}\right)=$ $\left\{(F(x z))^{\beta}:|x|=1\right\}$.

PROOF. Note that $F^{\beta}$ is analytic in $\Delta$ for some choice of $\log F(z)$. Also $(F(z))^{\beta}=K(z) /\left(z-x_{0}\right)^{\beta \alpha}$ where $K(z)=(G(z))^{\beta}$ is analytic in $\Delta$, continuous in $\bar{\Delta}, K\left(x_{0}\right) \neq 0$ and $\beta \alpha>1$. It is easy to deduce from (4) and the fact that $\log F(z)$ is convex that $E H s\left(F^{\beta}\right) \subset\left\{(F(x z))^{\beta}:|x|=1\right\}$. It follows that (4) holds with $F$ replaced by $F^{\beta}$. Hence the proof may be completed by appealing to Theorem 1.

We thank the referee for his suggestions and comments.

\section{REFERENCES}

1. Y. Abu-Muhanna, Variability regions and support points of subordinate families, J. London Math. Soc. (2) 29 (1984), $477-484$

2. $\_$, Subordination and extreme points, Complex Variables 9 (1987), 91-100.

3. L. Brickman, T. H. MacGregor and D. R. Wilken, Convex hulls of some classical families of univalent functions, Trans. Amer. Math. Soc. 156 (1971), 91-107.

4. P: C. Cochrane and T. H. MacGregor, Fréchet differentiable functionals and support points for families of analytic functions, Trans. Amer. Math. Soc. 236 (1978), 75-92.

5. D. J. Hallenbeck and T. H. MacGregor, Support points of families of analytic functions described by subordination, Trans. Amer. Math. Soc. 278 (1983), 523-545.

6. L Linear problems and convexity techniques in geometric function theory, Pitman, 1984.

7. O. Toeplitz, Die linearen volkommenen Räume der Funktionentheorie, Comment. Math. Helv. 23 (1949), 222242.

Department of Mathematical Sciences, University of Delaware, Newark, DELAWARE 19716 